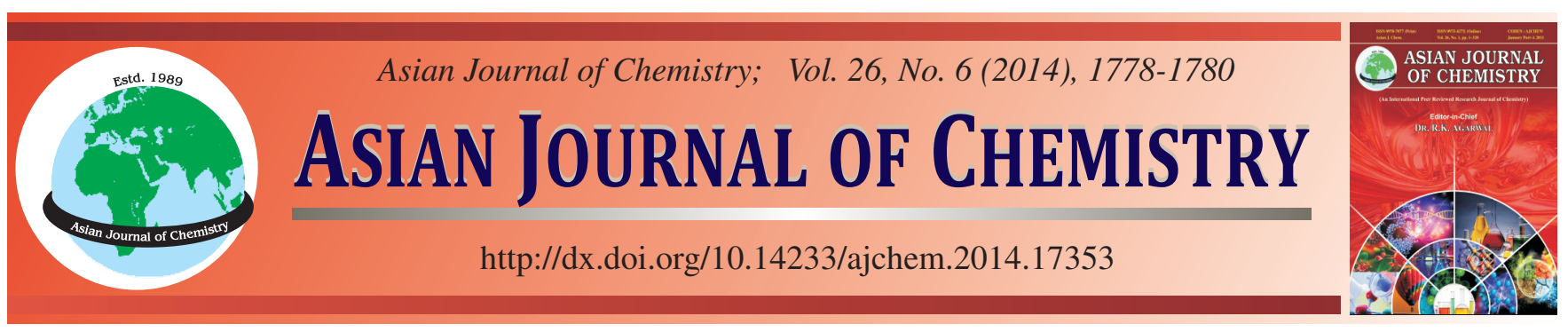

\title{
Morphologies, Mechanical Properties and Thermal Stability of Poly(lactic acid)/Waste Rubber Powder Blends†
}

\author{
Jinian YANG ${ }^{*}$, GuOXIN Ding, ZhoufEng WANG and ChuANG WANG
}

College of Materials Science and Engineering, Anhui University of Science and Technology, Huainan 232001, P.R. China

*Corresponding author: E-mail: yangjinian@163.com

\begin{abstract}
Poly(lactic acid) (PLA) blends containing varied mass fraction of waste rubber powder (WRP) were fabricated by melt compounding. Moreover, the morphologies, mechanical properties and thermal stability of the samples were investigated systematically. SEM observation shows that the well bonded interfacial structures between PLA and WRP particles are observed with the aid of silane coupling agent. Mechanical tests point out that the WRP could increase the impact toughness with the content up to $15 \%$. However, only a slight improvement in tensile strength when WRP content reaches $10 \%$ and the elastic modulus is decreased continuously with the rise of WRP. Thermogravimetric analysis shows the thermal decomposition temperatures of blends are declined gradually with WRP loading.
\end{abstract}

Keywords: Poly(lactic acid), Waste rubber powder, Toughen, Thermal stability.

\section{INTRODUCTION}

Poly(lactic acid) (PLA) which is characterized by excellent biodegradability and biocompatibility has obtained an extensive attention and applied widely in biomedical fields ${ }^{1}$. However, for the environmental aware, the fact that PLA derived from renewable plant resources makes it even more attractive to be one of the vast potential alternative to traditional petroleumbased polymers and its competitive strength and stiffness compared to those general resins would accelerate this process. Unfortunately, the poor impact resistance and high cost of PLA would restrict the wide applications in a way. A lot of efforts have been carried out and to introduce the elastomers has been confirm as the most effective method for improving the impact toughness ${ }^{2}$, but still seems not efficient for reducing the product price. Waste rubber powders (WRP) are organic particles primarily derived from waste tires and characterized by very low cost. Waste rubber powder has been utilized to improve the toughness in polypropylene $(\mathrm{PP})^{3,4}$ and polyethylene $(\mathrm{PE})^{5,6}$ and is also expected for enhanced the impact resistance of PLA. Therefore, in this paper, the PLA/WRP blends were prepared firstly and then the microstructures and properties were investigated.

\section{EXPERIMENTAL}

Poly(lactic acid) (REVODE101) was supplied by Hisun Biomaterials Co., Ltd, China. WRP was purchased from Hangzhou Baoli Recycling Co., Ltd., China. Silane coupling agent (A1100, A.R. grade) was the product of Nanjing Shuguang Chemical Co., Ltd. Absolute Alcohol and dimethyl silicone oil are both commercial available with A.R. grade.

Firstly, the WRP was treated with A1100 and then the PLA/WRP blends were prepared using melt-compounding and compression molding. The samples were consisted as follows: 100/0, 95/5, 90/10, 85/15, 80/20, 75/25, 70/30 (PLA/WRP, mass ratio).

The un-notched Charpy impact tests were performed by using a TCJ-25J impact tester according to ISO 179-1: 2000. Thermal stability was performed with a SDT2960 thermogravimetric analyzer (TGA) heated within the temperatures range $50-550{ }^{\circ} \mathrm{C}$ at a rate of $10{ }^{\circ} \mathrm{C} / \mathrm{min}$, under a controlled dry nitrogen flow of $60 \mathrm{~mL} / \mathrm{min}$. The impact fractured surfaces were coated with thin layer gold and observed with a Hitachi S-4800 field emission scanning electron microscopy (FESEM). 


\section{RESULTS AND DISCUSSION}

Morphologies of PLA/WRP blends: As is seen from Fig. 1, the WRP particles are dispersed in the matrix with irregular shapes and sizes (light colour areas), exhibiting the similar droplet-matrix structures. When content of WRP is not more than $15 \%$, the WRP particles dispersed uniformly and almost existed by way of single particle. However, the increased loading worsens the distribution of WRP. Fig. 1d shows great WRP aggregates being presented with $25 \%$ of WRP due to the foregone results in an incompatible system. Moreover, Fig. 1(b) also shows that there is no obvious interface de-bonding between the PLA matrix and WRP particles, which indicates the formation of close interfacial adhesion. This means that the pretreatment of WRP with A1100 is effective for improving the compatibility between WRP and PLA, as should be positive to enhance the performance of PLA/WRP blends.
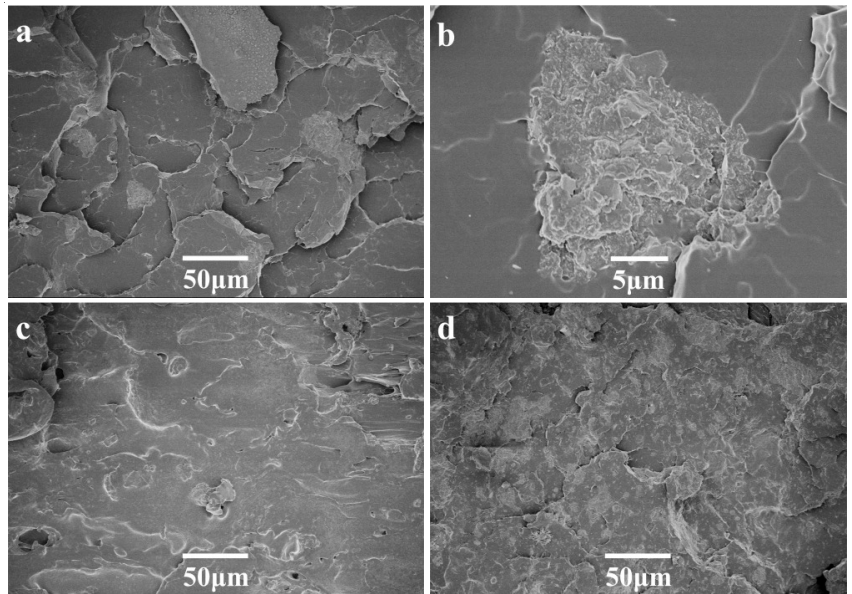

Fig. 1. Morphologies of impact fracture surfaces of PLA/WRP blends: (a) $5 \%$ WRP, (b) $5 \%$ WRP with high magnification, (c) $15 \%$ WRP, (d) $25 \% \mathrm{WRP}$

Impact toughness: Fig. 2 shows that the impact toughness was increased gradually until the peak value of $37.39 \mathrm{~kJ} / \mathrm{m}^{2}$ is obtained with $15 \%$ WRP, showing $158.4 \%$ higher than neat PLA. It should be attributed to the rubbery nature of WRP particles. When the samples are subjected to the high speed impact loading, the WRP particles should have the function in releasing the plastic constraint and inducing shear deformation of matrix, as well as be able to hinder or deflect the ways of crack propagation, thus more energy is consumed before materials crushed and increased the impact toughness. However, the sharply decline of impact toughness should be explained by the aggregation of excessive WRP particles acting as points of stress concentration, facilitating the failure process of the samples.

Tensile properties: Fig. 3 illustrates that the introduction of WRP just brought on a slight increase of the tensile strength, from 47.14 MPa for pure PLA to 48.57 MPa for PLA/WRP (90/10) blends and then decline sharply. Meanwhile, the elastic modulus exhibits a nearly linear decrease from $1.98 \mathrm{GPa}$ for PLA to $1.46 \mathrm{GPa}$ for blends with $30 \% \mathrm{WRP}$. There are two main factors, in this case, that might affect the tensile properties. On one hand, the WRP particles are possessed of much lower strength and stiffness than PLA and would induce a decrease

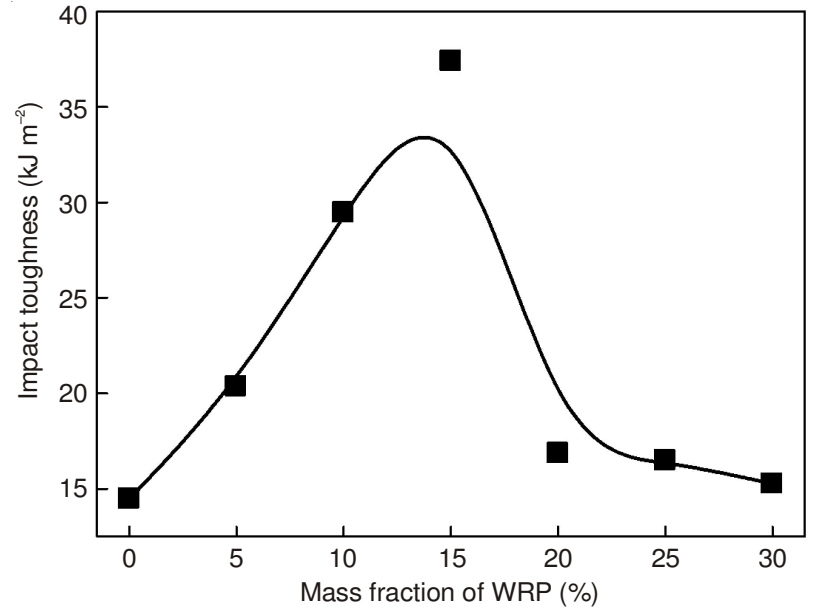

Fig. 2. Impact toughness of PLA/WRP blends

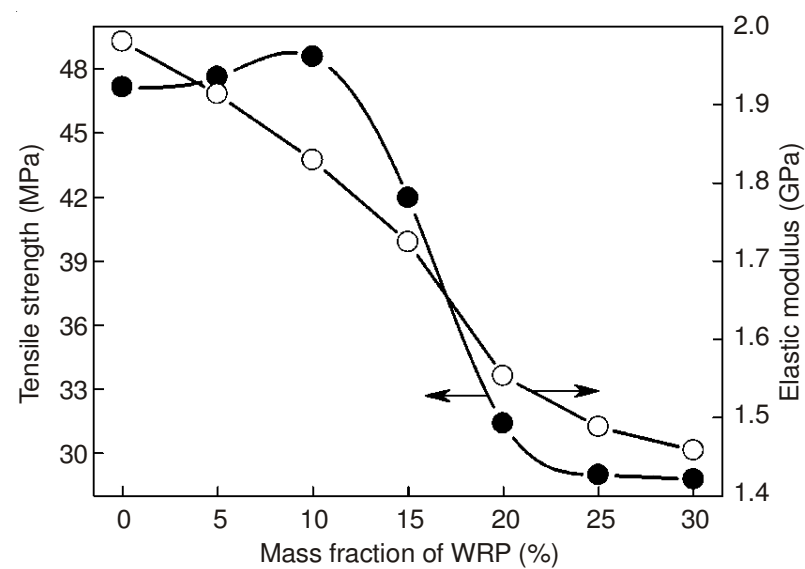

Fig. 3. Tensile strength and elastic modulus of PLA/WRP blends

in the strength and stiffness according to the rules of mixture. On the other hand, the WRP particles are very different from the normal rubber particles due to their inherent cross-linked structures. So, the WRP particles behave more like "natural" core-shell structures. This unique structure, together with the well bonded interfaces (Fig. 1), should somewhat restrain the plastic deformation when subjected to the quasi-static tensile load and showing a beneficial influence on strengthening the materials. When the loading of WRP particles is relative low (i.e., less than $10 \%$ ), the negative effect brought by the first factor would be conquered by the positive effect resulted from the second factor, then the tensile strength increases. However, the negative effect would behave increasingly dominant with the continuous rise of WRP. As a result, the tensile strength and elastic modulus of PLA/WRP blends are decreased.

Thermal stability: As could be seen from Fig. 4, the thermal degradations of neat PLA, WRP and their blends show the complete first-order loss model and exhibited the zigzaglike curves, indicating that the incorporation of WRP into PLA seems to have no influence on the thermal degradation model. The initial decomposition temperatures of the PLA/WRP blends are decreased gradually from $330.1{ }^{\circ} \mathrm{C}$ for pure PLA to the $297.7{ }^{\circ} \mathrm{C}$ for PLA/WRP $30 \%$, which should be due to the much lower initial decomposition temperature of WRP than pure PLA. However, the weight remaining percentages are increased steadily with WRP loading. This would be ascribed 


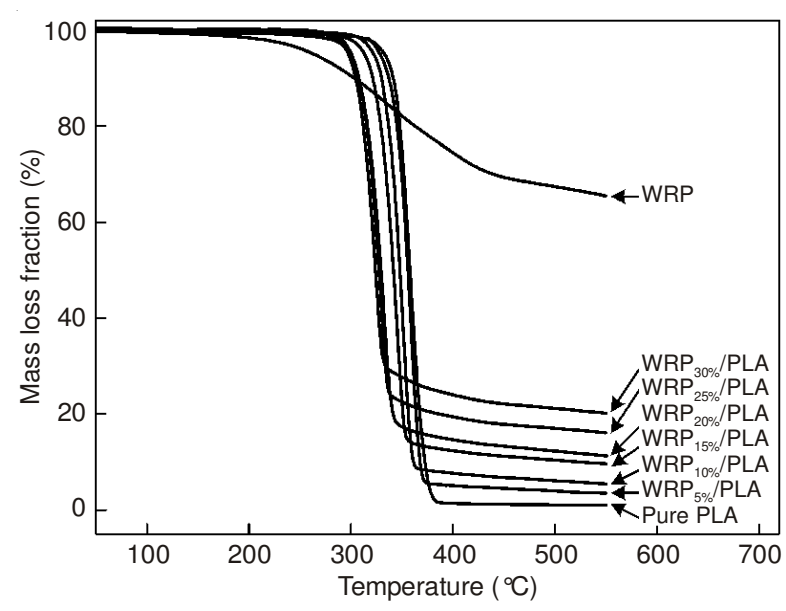

Fig. 4. Thermal stabilities of WRP, PLA and PLA/WRP blends

to the fillers, such as carbon black, used in WRP are stable at high temperatures and can not be removed by ignition under nitrogen, then acting as barriers to minimize the permeability of volatile degradation products in the blends.

\section{Conclusion}

This study mainly clarified the effects of WRP on the microstructures, impact toughness and thermal stability of PLA/WRP blends. FESEM observation shows the pretreatment of silane coupling agent could promote the compatilization of WRP and PLA in a way. The impact test presents that the WRP could toughen the PLA indeed with a $158.4 \%$ improvement when $15 \%$ WRP added. However, the tensile strength is only increased slightly and elastic modulus decreases gradually with the rise of WRP. TGA analysis indicates that the existence of WRP decreases the initial decomposition temperatures and increases the weight remaining percentages of PLA/WRP blends.

\section{ACKNOWLEDGEMENTS}

This work was supported by the Anhui University Provincial Natural Science Research Project (KJ2013Z067).

\section{REFERENCES}

1. K. Madhavan Nampoothiri, N.R. Nair and R.P. John, Bioresour. Technol., 101, 8493 (2010).

2. K.S. Anderson, K.M. Schreck and M.A. Hillmyer, Polym. Rev., 48, 85 (2008).

3. C.F. Ouyang, Q. Gao, Y.T. Shi and X.Q. Shan, J. Appl. Polym. Sci., 123, 485 (2012).

4. K.F. El-Nemr and A.M. Khalil, Addit. Technol., 17, 58 (2011).

5. Y. Li, Y. Zhang and Y.X. Zhang, Polym. Test., 23, 83 (2004).

6. S.H. Lee, Z.X. Zhang, D. Xu, D. Chung, J.G. Oh and J.K. Kim, Polym. Eng. Sci., 49, 168 (2009). 